\title{
SPORTS MEDICINE IN DISABILITIES
}

Ripari Patrizio ${ }^{1}$, Di Giacinto Gabriella ${ }^{2}$, di Sante Gianluca ${ }^{3}$, Barassi Giovanni ${ }^{4}$

${ }^{1}$ MD, Associate professor: Methods and didactics of motor activities, Department of Medical, Oral and Biotechnological Sciences, "G. d'Annunzio" University, Chieti-Pescara (Italy);

${ }^{2}$ MD, ASL1 Abruzzo - Avezzano-Sulmona, L'Aquila (Italy);

${ }^{3}$ Department of Medical, Oral and Biotechnological Sciences, "G. d'Annunzio" University, Chieti-Pescara (Italy); ${ }^{4} \mathrm{PhD}$, Coordinator of the Physiotherapy, Rehabilitation and Rehabilitation Centre (Ce.Fi.R.R.) Venue "G. d'Annunzio "University, Chieti-Pescara (Italy).

KEYWORDS: Sports, Exercise Therapy, Psychomotor Performance,

\section{ABSTRACT}

Daily physical activity is beneficial for all people and teenagers. For people with physical and sensorineural disabilities, similar recommendations regarding physical activity must be taken into account.

We hoped that the results of this contribution will promote our understanding of factors that influence physical activity levels and, in turn, inform about interventions to minimize inactivity.

Therefore, the purpose of this contribution is to examine the scientific literature to consider physical activity levels in sensorineural disabilities, observing how physical activity levels were measured and examining the reported factors that influenced the indications of the sports physician.

\section{INTRODUCTION}

Neurosensory and intellectual disabilities, also referred to as learning disabilities and/or intellectual motor development disorder are defined as "a significantly reduced ability to understand new or complex information and to learn and apply new skills, including motor skills" (World Health Organization, 2015).

Furthermore, to the extent that a subject is unable to meet the requirements established by society for the age group, it can be further classified as: mild, moderate, serious and profound (Salvador-Carulla et al., 2011; Katz and Lazcano-Ponce, 2008).

Prevalence estimates vary for several reasons, including methodological differences between studies (Doran et al., 2012; Maulik et al., 2011; Harris, 2006), the wealth of the country and the age group of the population studied (Harris , 2006). An analysis (Maulik et al., 2011) reported a prevalence of the population of 16.41 and $15.94 / 1000$ respectively in low and middle income countries, while in high income countries these figures are lower, with an estimated prevalence of the population of $9.21 / 1000$. In addition, they reported a higher prevalence among studies based on children / adolescents (18.30 / 1000), compared to those on adults (4.94 / 1000).

Importantly, these figures will increase due to the increase in life expectancy in this group of people (Emerson et al., 2014). This has implications since people with sensorineural and intellectual disabilities have poorer health than their non-disabled peers, with avoidable differences in health status (Heslop et al., 2014). These differences begin at the beginning of life, with a greater prevalence of diseases such as obesity, hypertension and hyperlipidemia, common among disabled people since adolescence (Lin et al., 2010)

In addition, interventions on exercise have shown that young people with physical disabilities can improve levels of physical well-being and reduce cardiometabolic risk factors (Verschuren et al., 2016; Vollins, 2010), normally having lower fitness levels participating less in competitive and recreational sports in general than in their fully developed peers.

\section{MATERIALS AND METHODS}

This study was conducted in accordance with the Meta-analysis guidelines of observational studies in epidemiology (MOOSE) for systematic reviews of observational studies (Stroup et al., 2000).

The studies eligible for the review included people with sensorineural disabilities and subjects with disabilities who competed and participated in sports for the disabled, without limitation for age, sex, sport or nature of the disability.

The initial search yielded 200 potentially relevant articles following the removal of duplicates. After the removal of studies that did not meet the title-based inclusion criteria, 40 articles remained. The abstracts of these articles were independently assessed by one of the authors, which further reduced the number of relevant studies to 25 . No studies were excluded from the full-text availability criteria. Finally the reference lists of the 25 articles were read, which identified five other studies.

\section{RESULTS}

Since limited physical capacity can interfere with physical activity in daily life and consequently affect their health later in life, maintaining sport participation and adequate fitness levels related to performance is particularly important in this population (Durstine et al., 2000).

For young people with physical disabilities, it seems more difficult to participate in sports and physical activities compared to fully develop peers (Maher et al., 2007). The reasons for this lower sporting participation are related not only to their physical 
disability but also to the lack of qualified support staff, to transportation problems, to the lack of acceptance and to the absence of nearby sports clubs. Most of these obstacles to physical activity can eventually be eliminated when a sports program is provided at school in the immediate hours following after-school. This setting offers a familiar environment with supported instructors, acceptance and no additional transportation except a delayed pickup from school (Jaarsma et al., 2015).

In addition, a study has proposed the need for initiatives at school level, as their integrative approach is effective and also addresses less active children. Recent work in the developing population has shown positive but inconsistent effects in increasing physical activity with post-school interventions (Pate et al., 2009). Interventions that lasted more than 12 weeks and focused exclusively on increasing physical activity by providing a sports program were the most effective. (Atkin et al., 2011).

According to the principles of exercise, a training frequency of at least three sessions per week is recommended to improve cardiorespiratory fitness in young people who are generally developing (Garber et al., 2011). Conversely, for children and adolescents with cerebral palsy who are highly deconditioned, two sessions per week are also enough to induce or maintain positive physical effects (Verschuren et al., 2016). On the other hand, from the point of view of parents, sport participation once a week is considered sufficient (Wiart et al., 2015). Parents must prioritize the frequency of sporting participation to the needs of daily life (Wiart et al., 2010). In a recent qualitative research, all parents indicated that the intensity or frequency of a sports program to improve fitness levels was not important to them. They stressed the importance of sport as "being active", having fun and socializing (Wiart et al., 2015).

To improve fitness levels, there is a discrepancy between exercise recommendations and feasibility in the daily life of young people with disabilities and their parents. Providing a sports program at school once a week can be implemented relatively easily and increase the level of sports participation of children. However, it is not known whether one session a week is beneficial for fitness and health in this population. A physically inactive lifestyle increases the risk of non-communicable diseases. However, increased physical activity has been shown to improve health outcomes (Richards et al., 2013).

Despite clear guidance on the need for an active lifestyle, several studies in the literature have reported the absence of regular physical activity in adults with intellectual disabilities (Finlayson et al., 2009; McGuire et al., 2007).

Compared to the general population, there are gaps in our knowledge of their physical activity. For example, individual factors such as gender, race and social status that have been shown in the general population to influence activity levels of people are actually not considered for the disabled people.

To realize effective programs of prevention of nontransmittable diseases, politicians needs clear data about the public assistance (Hallal et al., 2012). However, to date, no systematic review about that problem does exist. A review on activity levels in disabilities did not use a systematic methodology (Temple et al., 2006). This lack of literature suggest the importance of a systematic review regarding the activity level of disabled people, with the aim to identify most of the risk factors and increase the promotion of sport activity through the disabled population.

Participation in sport can help people with physical and sensorineural disabilities to gain self-esteem, promote the development of positive mental attitudes and help them come to terms with their disability and achieve social reintegration. Furthermore, it has been observed that people with disabilities with psychodepressive states manage to solve this aspect of their disability by being able to take part in sport (Bantjes et al., 2018).

Sport infuses self-discipline, competitive spirit and camaraderie. Its value in promoting health, physical strength, endurance, social offerings and psychological well-being are clear. It is not difficult to understand why sport is so important for the well-being of people with disabilities (Bendixen et al., 2014). In the past, the effects of sport on the rehabilitation of people with paraplegia and quadriplegia were studied, observing that sport activities allowed them to overcome boredom in the hospital and also to promote the development of physical and cardiorespiratory endurance (Schneider, 1966). For example wheelchair sports such as basketball help to develop coordination (Kjaer et al., 2000) where the disabled person has to push the wheelchair and learn to pass, grasp and grab the ball.

Therefore the sports doctor will then take care of preventing the usual complications of immobility and the treatment of injuries that may result from sports activities, indicating the right kind and level of physical activity. In fact some disabilities do not prevent participation but require specific precautions (Willis et al., 2018).

\section{CONCLUSIONS}

For many years disabled people showed interest for sport activities. Actually they have the opportunity to participate to many different sports. Many studies evaluated sport as an essential part of the management of patients affected by neurosensory pathology. The last five decades were characterized by a significant growing interest for sport for disabled people, not only from patients but also by doctor, sport associations and governments.

A large number of sport activities can be used for rehabilitation and recreation of disabled people. Sport is increasingly used as a complementary method to the classical physiotherapeutic approach, helping in developing physical strength, resistance and coordination.

However the severity of the disability can greatly influence the kind and quantity of sport activity that the patient can perform. It is clear for example that some sports, like fishing, flying kites and birdwatching, requires a limited physical effort, while other sports, like wheelchair basketball, driving and sailing, requires a far higher physical effort of strength and coordination.

In general, it is possible to affirm that people with disabilities should perform sports suitable for their mobility level. For example, paraplegic people prone to muscular spasm should consider particular prevention systems, like containment belts on the wheelchair, to avoid the possibility to fall from the 
wheelchair while performing the activity. At the same time it is clear that people affected by a complicated compresence of both physical and learning limitations require more supervision. Moreover, it should be taken into account that not all disabilities are static: some conditions, like multiple sclerosis, are very variable, while other pathologies, like muscular dystrophy, are progressively invalidating. It is thought that the level of activity of disabled people should be calibrated and constantly monitored during time, in accordance with the specific necessities of the patient in relation to his clinical condition.

Therefore, in conclusion, the role of the sport medicine doctor is considered to be very important in this field and his evaluation should be considered as important factors to verify the capacity of each patient to participate to a specific sport activity in the perspective of a multidisciplinary collaboration in the health field.

\section{REFERENCES}

1. Atkin, A. J., Gorely, T., Biddle, S. J. H., Cavill, N., \& Foster, C. (2011). Interventions to promote physical activity in young people conducted in the hours immediately after school: a systematic review. Int J Behav Med, 18, 176-187.

2. Bantjes, J., \& Swartz, L. (2018).Social Inclusion Through Para sport: A Critical Reflection on the Current State of Play. Phys Med Rehabil Clin N Am., 29(2), 409-416.

3. Bendixen, R. M., Lott, D. J., Senesac, C., Mathur, S., \& Vandenborne, K. (2014). Participation in daily life activities and its relationship to strength and functional measures in boys with Duchenne muscular dystrophy. Disabil Rehabil., 36(22), 1918-1923.

4. Collins, E. G., Gater, D., Kiratli, J., Butler, J., Hanson, K., \& Langbein, W. E. (2010). Energy cost of physical activities in persons with spinal cord injury. Med Sci Sports Exerc, 42, 691-700.

5. Doran, C. M., Einfeld, S. L., \& Madden, R. H. (2012). How much does intellectual disability really cost? First estimates for Australia. J. Intellect. Develop. Disabil., 37(1), 42-49.

6. Durstine, J. L., Painter, P., Franklin, B. A., Morgan, D., Pitetti, K. H., \& Roberts, S. O. (2000). Physical activity for the chronically ill and disabled. Sports Med, 30, 207-219.

7. Emerson E., Glover G., Hatton C., \& Wolstenholme J. (2014). Trends in age-standardised mortality rates and life expectancy of people with learning disabilities in Sheffield over a 33-year period. Tizard Learn. Disabil. Rev., 19(2), 90-95.

8. Finlayson J., Jackson A., \& Cooper S. A. (2009). Understanding predictors of low physical activity in adults with intellectual disabilities. J. Appl. Res. Intellect. Disabil., 22(3), 236-247.

9. Garber, C. E., Blissmer, B., Deschenes, M. R., Franklin, B. A., Lamonte, M. J., Lee, I. M., et al. (2011). American college of sports medicine position stand. Quantity and quality of exercise for developing and maintaining cardiorespiratory, musculoskeletal, and neuromotor fitness in apparently healthy adults: guidance for prescribing exercise. Med Sci Sports Exerc, 43, 1334-1359.

10. Hallal, P. C., Andersen, L. B., Bull, F. C., Guthold, R., Haskell, W., \& Ekelund, U. (2012). Global physical activity levels: surveillance progress, pitfalls, and prospects. Lancet, 380(9838), 247-257.

11. Harris, J. C. (2006). Intellectual disability: Understanding its development, causes, classification, evaluation, and treatment. Oxford University Press.

12. Heslop, P., Blair, P. S., Fleming, P., Hoghton, M., Marriott, A., \& Russ, L. (2014). The confidential inquiry into premature deaths of people with intellectual disabilities in the UK: a population-based study. Lancet, 383(9920), 889-889.

13. Jaarsma, E. A, Dijkstra, P. U., de Blécourt, A. C. E., Geertzen, J. H. B., \& Dekker, R. (2015). Barriers and facilitators of sports in children with physical disabilities: a mixed-method study. Disabil Rehabil, 37, 1617-1625.

14. Katz, G., \& Lazcano-Ponce, E. (2008). Intellectual disability: definition, etiological factors, classification, diagnosis, treatment and prognosis. Salud Publica Mex., 50.

15. Kjaer M. (2000). Why exercise in paraplegia? Br J Sports Med., 34(5), 322-323.

16. Lin, P. Y., Lin, L. P., \& Lin, J. D. (2010). Hypertension, hyperglycemia, and hyperlipemia among adolescents with intellectual disabilities. Res. Dev. Disabil., 31(2), 545-550.

17. Maher, C. A., Williams, M. T., Olds, T., \& Lane, A. E. (2007). Physical and sedentary activity in adolescents with cerebral palsy. Dev Med Child Neurol, 49, 450-457.

18. Maulik, P. K., Mascarenhas, M. N., Mathers, C. D., Dua, T., \& Saxena, S. (2011). Prevalence of intellectual disability: a metaanalysis of population-based studies. Res. Dev. Disabil., 32(2), 419-436.

19. McGuire, B. E., Daly, P., \& Smyth, F. (2007). Lifestyle and health behaviours of adults with an intellectual disability. J. Intellect. Disabil. Res., 51(7), 497-510

20. Murphy, N., \& Carbone, P. (2008). American Academy of Pediatrics Council on Children With Disabilities. Promoting the participation of children with disabilities in sports, recreation, and physical activities. Pediatrics, 121, 1057-1061.

21. Pate, R. R., \& O'Neill, J. R. (2009). After-school interventions to increase physical activity among youth. Br J Sports Med, 43, 14-18.

22. Richards, J., Hillsdon, M., Thorogood, M., \& Foster, C. (2013). Face-to-face interventions for promoting physical activity. Cochrane Database Syst. Rev., 9.

23. Salvador-Carulla, L., Reed, G. M., \& Vaez-Azizi, L. M. (2011). Intellectual developmental disorders: towards a new name, definition and framework for "mental retardation/intellectual disability" in ICD-11. World Psychiatry, 10(3), 175.

24. Schneider, G. (1966). The value of sport as a recreation in paraplegia. Aust J Physiother, 12(2), 62-66.

25. Stroup, D. F., Berlin, J. A., Morton, S. C., et al. Meta-analysis of observational studies in epidemiology: a proposal for reporting. JAMA, 283, 2008-2012.

26. Temple, V. A., Frey, G. C., \& Stanish, H. I. (2006). Physical activity of adults with mental retardation: review and research needs. Am. J. Health Promot., 21(1), 2-12.

27. Verschuren, O., Peterson, M., Balemans, A., \& Hurvitz, E. (2016). Exercise and physical activity recommendations for people with cerebral palsy. Dev Med Child Neurol, 58, 798-808.

28. Wiart, L., Darrah, J., Kelly, M., \& Legg, D. (2015). Community fitness programs: what is available for children and youth with motor disabilities and what do parents want? Phys Occup Ther Pediatr, 35, 73-87.

29. Wiart, L., Ray, L., Darrah, J., \& Magill-Evans, J. (2010). Parents' perspectives on occupational therapy and physical therapy goals for children with cerebral palsy. Disabil Rehabil, 32, 248-258.

30. Willis, C. E., Reid, S., Elliott, C., Rosenberg, M., Nyquist, A., Jahnsen, R., \& Girdler, S. (2018). A realist evaluation of a physical activity participation intervention for children and youth with disabilities: what works, for whom, in what circumstances, and how? BMC Pediatr., 18(1), 113.

31. World Health Organization. (2015). Definition: intellectual disability. Copenhagen (Denmark): Regional Office for Europe. 